\title{
Los murciélagos de La Bóveda (Segovia), treinta y dos años después
}

\author{
FÉlix González Álvarez ${ }^{1}$, Óscar de Paz² y Montserrat Carbonell ${ }^{3}$
}

\author{
'Grupo Asturiano para el Estudio y Conservación de los Murciélagos. Calle carrión, 14-3 H. E-33424, \\ Posada de Llanera. Principado de Asturias. España. \\ ${ }^{2}$ Departamento de Zoología y Antropología Física, Universidad de Alcalá. E-28871 \\ Alcalá de Henares, Madrid, España. \\ ${ }^{3} \mathrm{Ch}$. des Grands-Champs 5, 1874, Champéry, Suiza.
}

Correo electrónico del autor: fei2@ctv.es

DOI: http://dx.doi.org/10.14709/BarbJ.5.1.2012.06

English title: The bats of La Bóveda (Segovia) thirty-two years on

\begin{abstract}
La Bóveda is an underground corridor in the Royal Palace of La Granja, Segovia. In 1976-1978 its bat population was monitored and 32 years later this monitoring has been repeated. During this period of time all the entrances to the underground corridor were closed, although two entrances were opened again some years after being closed off. Nevertheless, the bat populations have not recovered to the levels present in the 1970s. Currently, the roost is used by six of the 10 species that occupied this shelter in the 1970s and continues to host a large group of Myotis escalerai (500 adults). Barbastella barbastellus and Miniopterus schreibersii have not returned to the Royal Palace, although they have been observed roosting in the vicinity at a distance of less than $500 \mathrm{~m}$.
\end{abstract}

Keywords: La Bóveda, Palacio Real de la Granja, Segovia, roosts, conservation, Myotis escalerai, Miniopterus schreibersii, bats.

Resumen: La Bóveda es un conducto subterráneo de los jardines del Palacio Real de La Granja, en Segovia. Durante los años 1976 a 1978 su población de murciélagos fue objeto de un detallado seguimiento que ha vuelto a ser repetido 32 años después. En este período de tiempo, los accesos a la galería fueron cerrados, abriéndose dos de las entradas varios años después, aunque no se ha conseguido revertir la situación a la de los años setenta. En la actualidad la galería es utilizada por seis de las diez especies que lo ocupaban en los años setenta y continúa albergando una importante agrupación de $M$. escalerai (unos 500 adultos). Sin embargo, B. barbastellus y $M$. schreibersii no han vuelto a ser localizados en la galería, aunque si en otros refugios muy próximos (distancia inferior a $500 \mathrm{~m}$ ).

Palabras clave: La Bóveda, Palacio Real de la Granja, Segovia, refugios, conservación, Myotis escalerai, Miniopterus schreibersii, murciélagos.

\section{INTRODUCCIÓN}

La información sobre seguimientos detallados de refugios de murciélagos en Europa prácticamente se halla limitada a países del centro y norte del continente, sobre todo durante la época invernal (por ejemplo, Degn 1987; Fuszara et al. 1996; Furmankiewicz \& Górniak 2002; Višňovská et al. 2006; Masing et al. 2009). Por el contrario, es anecdótica en países mediterráneos, en los que sólo se dispone de datos de algunas visitas en diferentes años (Dragu 2009) y para ciertas especies (Ramos et al. 2009).

Durante 1976, 1977 y 1978, se realizó en La Bóveda un estudio sobre la composición específica y las variaciones numéricas en la presencia de las diferentes especies de murciélagos (Carbonell 1979a). A pesar de tratarse del trabajo de seguimiento más detallado realizado en la época y uno de los primeros que se realizó en España, sus resultados sólo fueron parcialmente publicados (Carbonell 1979b). Gracias al Consejo de Administración de Patrimonio Nacional (Ministerio de la Presidencia), durante los años 2009 y 2010 se pudo realizar un estudio similar en el mismo lugar (Paz \& González-Álvarez 2010), permitiendo comparar las observaciones realizadas en ambos períodos.

\section{MATERIALES Y MÉTODOS}

«La Bóveda» (figura 1) es el nombre por el que se conoce a la mayor galería por la que se canaliza el abastecimiento de agua de varias fuentes de los jardines del Palacio Real de La Granja, en la localidad de San Ildefonso, en Segovia. Este conducto, de unos $300 \mathrm{~m}$ de longitud, $3 \mathrm{~m}$ de anchura y entre 1,7 y $2,9 \mathrm{~m}$ de altura, fue excavado cuando se iniciaron las obras de construcción de los jardines, a principios del siglo XVIII, y ha permanecido prácticamente en su estado original hasta nuestros días. 
La galería se halla comunicada con el exterior a través de once troneras de sección cuadrada y cerca de un metro de lado. Tres troneras más (XII a XIV) situadas al final de la galería no tienen comunicación con el exterior. Durante el estudio de los años 1976-1978 las troneras estaban abiertas, sin obstáculos y sin otra protección que el muro que sobresale del terreno. A finales de los años ochenta se colocaron rejas de metal en todas ellas como medida de seguridad para los visitantes de los jardines. En el año 2000, gracias al programa Life/Naturaleza 96NAT/E/003081 (“Actuaciones prioritarias para la protección de los quirópteros en zonas de interés comunitario de Castilla y León"), se dejaron permanentemente abiertas las rejas de dos troneras (VI y VII), manteniendo el resto cerradas (Benzal 2000).

En el estudio de los años setenta La Bóveda fue visitada semanalmente desde octubre de 1976 hasta diciembre de 1977. Durante 1978 las visitas se realizaron una vez al mes. En el trabajo desarrollado en 2009-2010 el seguimiento se ha realizado visitando la galería una vez al mes entre abril de 2009 y el mismo mes del año 2010. En ambos casos se realizó una detallada inspección visual, registrando información tanto sobre la composición específica y numérica de las especies de murciélagos localizadas como de la ubicación espacial de los ejemplares. En el periodo 2009-2010 se capturó una pequeña muestra de ejemplares mediante una manga quiropterológica con el fin de realizar análisis moleculares para la identificación de las especies.

Los censos, realizados en los años 1976-1978 por una persona y en los años 2009-2010 por dos, se llevaron a cabo mediante conteo directo, aunque en el seguimiento de 2009-2010 el número de individuos en los grupos se estimó a partir de fotografías. Para registrar la actividad en todos los accesos a la galería, entre mayo y septiembre de 2009 se realizaron grabaciones en vídeo infrarrojo con un detector de ultrasonidos, utilizándose también sensores pasivos de movimiento. Entre los meses de agosto de 2009 y abril de 2010 se colocó una cámara fotográfica automática programada para efectuar una fotografía cada 24 horas (sobre las 10 horas UTC) de la zona en la que se ubica el grupo más numeroso de murciélagos.

\section{RESUltados}

De las diez especies de quirópteros identificadas en 19761978, sólo seis fueron también observadas en 2009-2010 (tabla 1).

Tabla 1. Especies de murciélagos identificadas ( ) en los estudios realizados en la galería de La Bóveda del Palacio Real de la Granja de San Ildefonso durante los períodos 1976-1978 y 2009-2010.

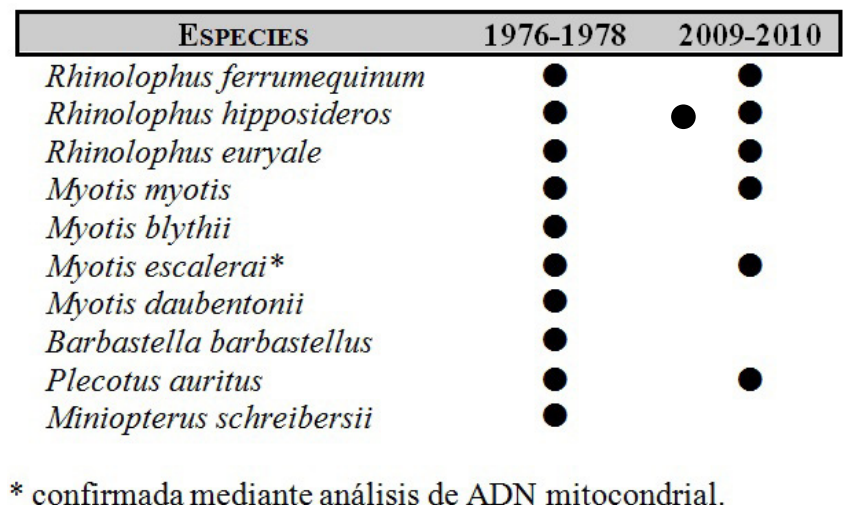

Los valores medios de los índices de diversidad de Shannon y de equitabilidad, considerando las observaciones registradas en visitas mensuales y en la misma secuencia temporal (abril a febrero), fueron mayores en 1976-1978 $(\mathrm{H}=0,499 ; \mathrm{J}=0,390)$ que en $2009-2010(\mathrm{H}=0,225 ; \mathrm{J}=0,199)$ (Test de Mann-Whitney: $U=0,97 ; \mathrm{N}=11 ; \mathrm{p}=0,008$. $\mathrm{Y} \mathrm{U}=0,94$; $\mathrm{N}=11 ; \mathrm{p}=0,014)$. Las mayores agrupaciones de ejemplares corresponden a $M$. escalerai (19/06/2009) (figura 2) y a $M$. schreibersii (26/08/1977), en los dos casos con algo más de 500 ejemplares. En ocho de las diez especies detectadas en 1976-1978 fueron observados dos o más ejemplares en una misma fecha, mientras que en 2009-2010 sólo lo fueron en dos, $R$. ferrumequinum y $M$. escalerai. Para estas dos especies el número de individuos observado en las visitas

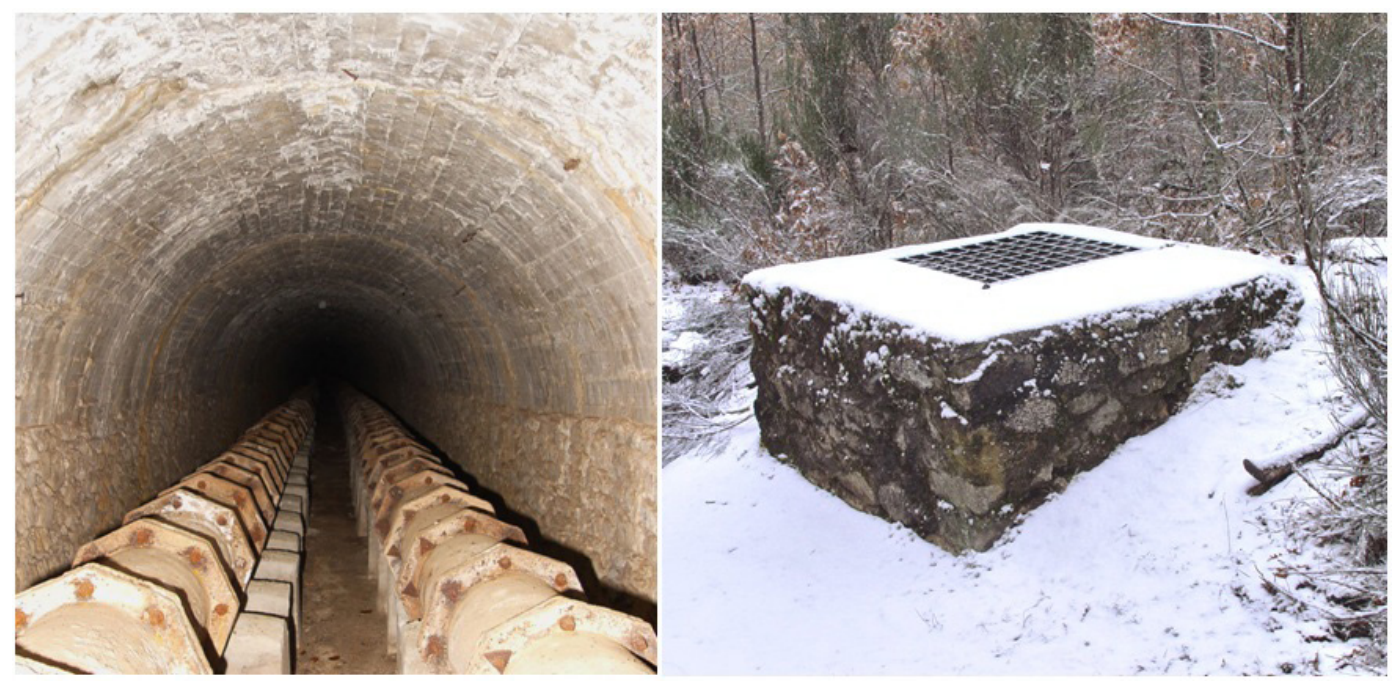

Fig. 1 Interior de La Bóveda y vista de una de las troneras, cerradas con rejas, que servían de acceso a los murciélagos.

(Fotografías de los autores) 
efectuadas en 2009-2010 es superior al registrado hace 30 años para la misma secuencia temporal (Test de Wilcoxon para muestras apareadas: $M$. escalerai: $\mathrm{T}<0,0001 ; \mathrm{N}=11$; $\mathrm{p}=0,001$. $R$. ferrumequinum: $\mathrm{T}<0,0001 ; \mathrm{N}=10 ; \mathrm{p}=0,002$ ). Ambas especies fueron localizadas durante todo el ciclo anual (ver en figura 3), tanto en 1976-1978 como en 20092010. De $R$. ferrumequinum casi siempre se observaron unos pocos ejemplares aislados, mientras que el número de M. escalerai fue variable a lo largo del año, registrándose ligeras diferencias entre ambos estudios: en 1976-1978 la especie fue más abundante desde principios del mes de julio hasta mediados de diciembre, siendo todos los ejemplares capturados (718) machos; mientras que en 2009-2010 el mayor número se registraba desde abril hasta octubre, detectándose la presencia de hembras (11 sobre una muestra de 26 ejemplares capturados) y crías en las inspecciones realizadas el 19 de junio y el 20 de julio. La presencia actual del resto de las especies es mas reducida que en 1976-1978 y entre todas ellas no superan los 25 ejemplares para todo el período de estudio. En el caso de R. euryale sólo se realizó

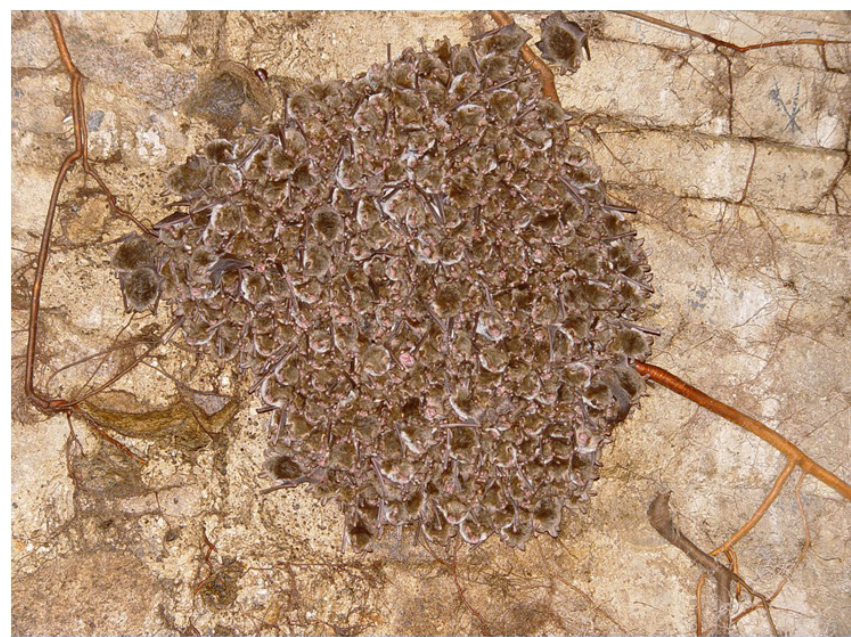

Fig. 2. En la actualidad La Bóveda es el refugio en el que se concentra la mayor agrupación de M. escalerai (Quétglas 2008). (Fotografías de los autores) una observación de un ejemplar solitario.
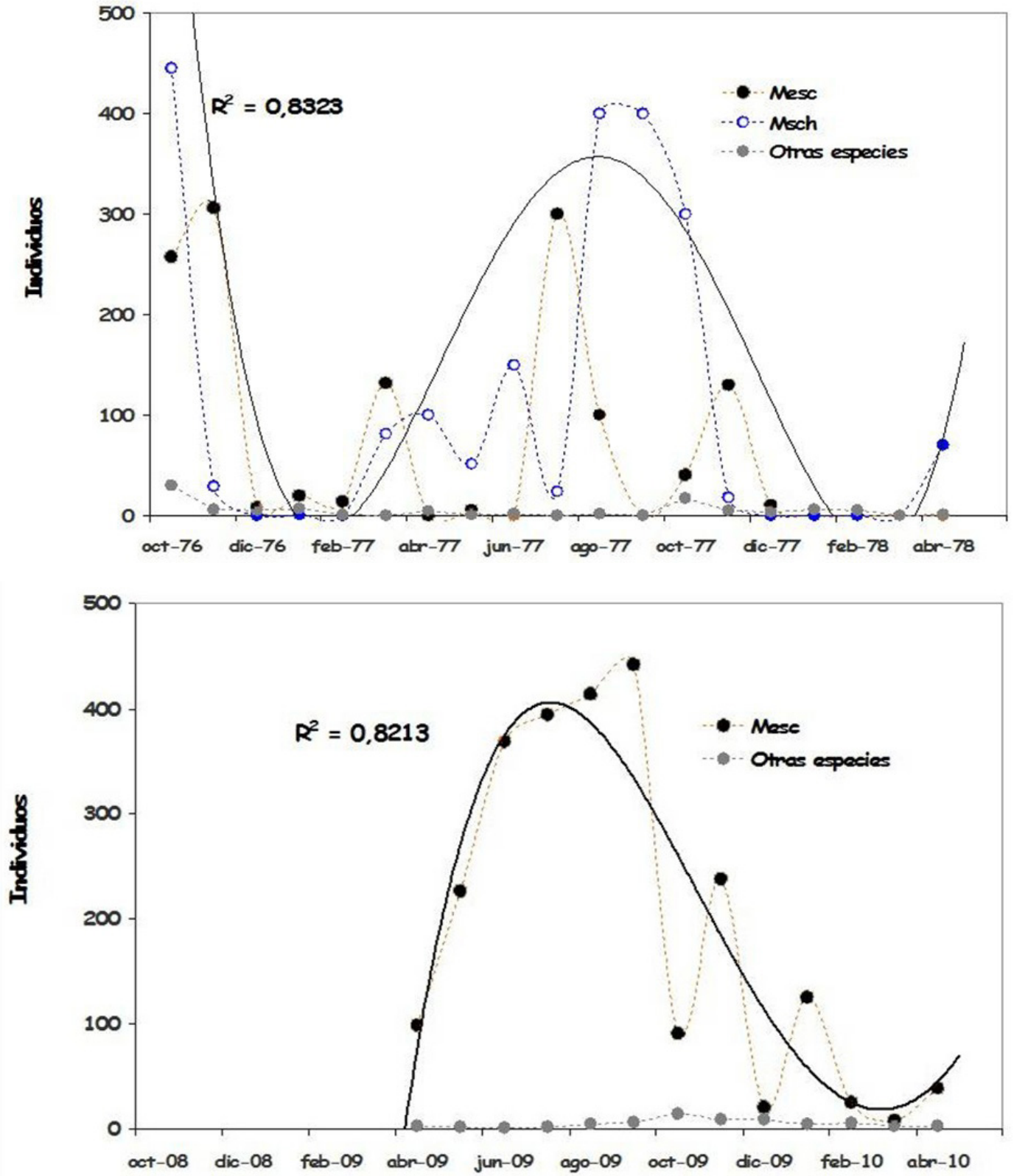

Fig. 3. Fluctuaciones numéricas de la presencia de murciélagos en La Bóveda en 1976-1978 (arriba) y 2009-2010 (abajo). Con trazo negro se representa la línea de tendencia polinomial ajustada (orden 4) para todas las especies. 

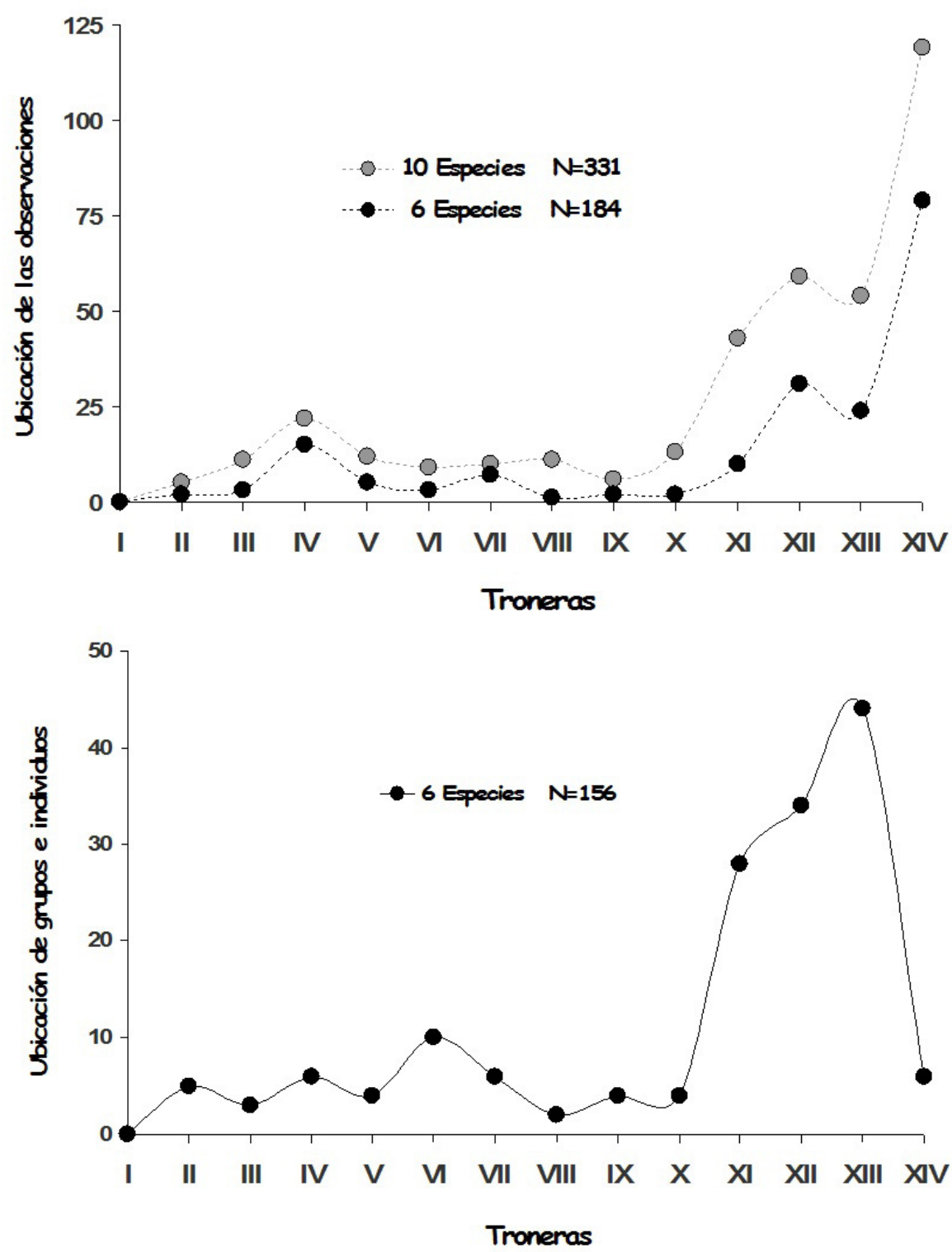

Fig. 4. Localización de las observaciones de murciélagos en diferentes tramos de la galería de La Bóveda en 1976-1978 (arriba) y 2009-2010 (abajo). En el eje de ordenadas se indica la situación de las observaciones según su posición respecto a las troneras. Cada observación corresponde a una localización, independientemente del número de ejemplares de la especie en ese emplazamiento.

Las diferencias registradas en la distribución espacial de los ejemplares en La Bóveda entre 1976-1978 y 2009-2010 no muestran diferencias significativas (Test de Wilcoxon para muestras apareadas: $\mathrm{T}=25,0 ; \mathrm{N}=12 ; \mathrm{p}=0,292$ ). Los murciélagos se localizan a lo largo de toda la galería, pero las mayores agrupaciones se sitúan siempre al final, entre las troneras XI y XIV (figura 4). La mayor parte de las observaciones de $M$. escalerai se realizaron siempre en una zona muy concreta próxima a la tronera XIII, en la que se situaba la colonia con cambios de emplazamiento casi diarios. Durante la época invernal (diciembre a marzo) los ejemplares de esta especie fueron localizados dispersos en la mayor parte de la galería, aislados o formando pequeñas agrupaciones en el interior de grietas de las paredes. Para $R$. ferrumequinum las observaciones fueron también más frecuentes en el último tramo de la galería, entre las troneras XI y XIV, localizándose algunos individuos aislados entre las troneras III y VII.
Las grabaciones de vídeo y los sensores de movimiento instalados confirman que en la actualidad la salida de los ejemplares de la galería se realiza exclusivamente por las dos troneras que permanecen abiertas y nunca por las troneras con rejas.

\section{Discusión}

La información sobre seguimientos durante un ciclo anual completo de refugios de murciélagos en España es sorprendentemente escasa (Carbonell 1979a, Paz 1985), sin que en ningún caso y tras varias décadas, se hayan repetido este tipo de investigaciones en los mismos refugios. Esta situación y la escasez de datos sobre la evolución de las colonias de murciélagos en España otorgan un particular interés al caso de La Bóveda.

Tanto en 1976-1978 como en 2009-2010, la mayoría de los murciélagos se localizaron en la parte final de la galería, en donde las temperaturas son más suaves, la humedad más 
elevada y las oscilaciones diarias menos acusadas (autores, datos inéditos). Las únicas excepciones fueron P. auritus y B. barbastellus, que nunca fueron observados en esta zona. Salvo para $R$. euryale, el patrón de distribución temporal del número de individuos para las especies localizadas en ambos períodos es similar, con una fuerte dominancia de M. escalerai. La presencia de M. daubentonii en la galería parece simplemente accidental ya que a pesar de localizarse con cierta abundancia en los jardines (Paz \& GonzálezÁlvarez 2010), sólo se observó un individuo en 1976.

La apertura de dos de las troneras cerradas no parece haber tenido los efectos de recolonización esperados, siendo la riqueza de especies inferior en la actualidad respecto a la registrada en 1976-1978. Dado que no se aprecian variaciones importantes en el estado de conservación del refugio, ni en sus condiciones microclimáticas, sólo los cierres instalados en las troneras (Benzal 2000) parecen constituir el principal factor que afectó a la calidad del lugar para las especies que no han sido detectadas en 2009-2010 (M. blythii, B. barbastellus y M. schreibersii), o sobre R. euryale y M. myotis, cuya presencia en la galería es considerablemente inferior a la registrada en 1976-1978. De acuerdo con los datos recogidos entonces para estas especies, La Bóveda tenía interés como refugio invernal de B. barbastellus y, sobre todo, como refugio de migración de M. schreibersii (Fernández Gutiérrez 2002), que formaba colonias equinocciales de otoño superiores a los 600 ejemplares. M. schreibersii es conocida por la dificultad para acceder a refugios con rejas no adaptadas especialmente para su acceso (Rodrigues \& Palmeirim 1996, Lumsden 1998 citado por Swifft 2010, González Álvarez \& Rodríguez-Muñoz 2003) y, de hecho, en los jardines de La Granja se refugia en otras galerías en las que no se han instalado verjas. En el caso de B. barbastellus no existen referencias tan claras sobre la incidencia de la instalación de obstáculos en la entrada a sus refugios (Baranauskas 2006), aunque en otros refugios subterráneos su presencia parece que podría estar influenciada por la existencia de variaciones climáticas exteriores (Masing et al. 2009). Ambas especies han sido localizadas en los jardines de La Granja en 2009 y 2010 durante la realización de otros trabajos de muestreo de murciélagos (Paz \& González-Álvarez 2010).

Además de la pérdida de biodiversidad en La Bóveda, es interesante señalar la observación de hembras y crías de $M$. escalerai en 2009, ya que no habían sido detectadas en 19761978, ni en otros trabajos (Benzal 2000).

En la actualidad, La Bóveda se muestra como un refugio muy estable para M. escalerai, siendo un lugar de hibernación para $R$. ferrumequinum. Durante el invierno es cuando se observa una menor presencia de murciélagos, pero tanto en esta época como durante el otoño se registra el mayor número de especies, localizándose ocasionalmente M. myotis, P. auritus, $R$. euryale y $R$. hipposideros. Numéricamente se trata del refugio en el que se concentraría la mayor agrupación de ejemplares adultos de $M$. escalerai citada en España (Quétglas 2008). Para el resto de las especies el número de individuos observado en 2009-2010 no es importante, como tampoco lo era antaño (Carbonell 1979a).

\section{REFERENCIAS}

Baranauskas, K., 2006. Bat species composition and abundance in two underground hibernaculae in Vilnius before and after fencing. Ekologija, 1: 1015 .

Benzal, J. 2000. La Bóveda de los Jardines de La Granja como refugio de murciélagos. Actuaciones llevadas a cabo para favorecer la recuperación de la colonia que albergaba. Consejería de Medio Ambiente de Castilla y León-CSIC. Proyecto LIFE 96NAT/E/003081: Actuaciones prioritarias para la protección de quirópteros en zonas de interés comunitario de Castilla y León. Valladolid. Informe inédito. $77 \mathrm{pp}$.

CARbonell, M., 1979a. Estudio de las colonias de quirópteros de La Bóveda en San Ildefonso. Segovia. Tesis de Licenciatura, Universidad Complutense de Madrid, 57 pp.

Carbonell, M., 1979b. Anillamientos y controles de quirópteros obtenidos en La Bóveda, en La Granja de San Ildefonso. Bol. Est. Centr. Ecol., 16: 67-71.

DeGn, H. J., 1987. Bat counts in Monsted Limestone cave during the year. Myotis, 25:85-90.

Dragu, A., 2009. Species structure of the bat community hibernating in Muierilor Cave (Southern Carpathians, Romania). North-Western Journal of Zoology, 5 (2): 281-289.

FERNÁNDEZ GUTIÉRREZ, J., 2002. Los murciélagos en Castilla y León. Atlas de distribución y tamaño de las poblaciones. Consejería de Medio Ambiente. Junta de Castilla y León, Valladolid.

FurmankiEWICZ \& GórniaK, 2002. Seasonal changes in number and diversity of bat species (Chiroptera) in the Stolec mine (SW Poland). Przyroda Sudetów Zachodnich Suplement, 2: 49-70

Fuszara E., M. Kowalski, G. LesińsKi \& J. P. Cygan, 1996. Hibernation of bats in underground shelters of central and northeastern Poland. Bonn. Zool. Beitr. 46: 349-358.

GonZÁlez Álvarez \& RodríGUEZ-MuÑoz, 2003. Protección de un refugio de hibernación del murciélago de cueva, Miniopterus schreibersii (Kuh, 1817), en Asturias. Pp. 42. En: II Jornadas sobre estudio y conservación de los murciélagos. Generalitat Valenciana-SECEMU. Valencia, 6-8 diciembre, 2003.

Masing, M., K. Baranauskas, Y. Sirvonenc \& T. WERMUNDSEN, 2009. Bats hibernating in Kaunas Fortress, Lithuania. Estonian Journal of Ecology, 58 (3): 192-204. DOI: https://dx.doi.org/10.3176/ eco.2009.3.04 
PAz, Ó. DE., 1985. Contribución al estudio eco-etológico de los quirópteros cavernícolas de "La Canaleja", Abanades. Guadalajara. Bol. Est. Central Ecol., 27:77-87.

PAz, Ó DE \& F. GonZÁlez-ÁlvAREZ, 2010. Plan de actuaciones para la conservación y manejo de murciélagos en distintas propiedades de Patrimonio Nacional, en La Granja y Aranjuez. MYOTIS C.B. TRAGSEGA. Patrimonio Nacional, Ministerio de la Presidencia (informe inédito), Madrid.

QuETGLAS, J.2008. Murciélago ratonero gris-Myotis nattereri. En: Enciclopedia Virtual de los Vertebrados Españoles. Carrascal, L. M., Salvador, A. (Eds.). Museo Nacional de Ciencias Naturales, Madrid. http://www. vertebradosibericos.org/. [10/08/2011].

Ramos M. J., P. Salgueiro, L. Rodrigues, M. M. Coelho \& J. M. Palmeirim, 2009. Population Structure of a Cave-Dwelling Bat, Miniopterus schreibersii: Does It Reflect History and Social Organization? Journal of Heredity, 100(5): 533-544. DOI: https://dx.doi. org/10.1093/jhered/esp032

Rodrigues, L. \& J. Palmeirim, 1996. Availação do impacto do encerramento de cavidades para protecção de colónias de morcegos cavernícolas. Instituto da Conservação da Naturaleza/Programa Life . Lisboa.

SwIFTT, 2010. Threatened mammals. Common Bent-wing Bat. http://bird.net.au/bird/index.php?title=Common Bent-wing_Bat. [18/10/2010].

VIŠŇovská, Z., J. Zelinka \& K. Strug 2006. Spatial distribution of hibernating bats (Chiroptera) in relation to climatic conditions in the Demänovská Ice Cave (Slovakia). Pp. 87-97. In: Proceedings of the 2nd International Workshop on Ice Caves, Demänovská Dolina, May 8-12, 2006.

\section{Agradecimientos}

Quisiéramos expresar nuestro agradecimiento a los gestores y, especialmente, a los guardas del Palacio Real de La Granja de San Ildefonso, que nos facilitaron su ayuda con una profesionalidad digna de mención y fueron un ejemplo de amabilidad y colaboración, incluso en aquellos días en los que la investigación y la conservación no eran parte del lenguaje diario en España. También queremos agradecer a los técnicos de Patrimonio Nacional (A. Jacoste y M. Moreno) y de la empresa TRAGSEGA (A. Rosón, M. Pérez y R. Martínez), por su confianza y su eficaz gestión. A J. M. Rey, y a P. Ceballos, E. Morales-Agacino y J. Molina, del extinto ICONA; a E. Ortíz, director del Museo Nacional de Ciencias Naturales de Madrid; y a J. Zamarro y J.Benzal por su ayuda en muchos aspectos. A R. Stebbings por su apoyo y transmitirnos su pasión por los murciélagos. A J. O. Carbonell y J. Carbonell, M. J. Moreno, J. de Lucas y B. del Horno por su siempre valiosa colaboración en todas aquellas salidas de campo en las que nos acompañaron. Y a Eduardo J. González, que facilitó la confección de la topografía de La Bóveda y nos aconsejó en diversas cuestiones técnicas. I. Garín aportó sugerencias críticas al manuscrito.

El estudio realizado en 2009-2010 ha sido financiado por Patrimonio Nacional a través de la empresa Tragsa y gracias a la mediación de la Sociedad Española para la Conservación y Estudio de los Murciélagos (SECEMU). Para su realización se contó con la autorización para la captura y manejo de murciélagos de la Junta de Castilla y León (EP/SG/352/2009). 